\title{
Online-Datenbank
}

\section{Transparenz bei Antibiotika-Resistenzen}

\author{
Wollen Ärzte wissen, wie es mit Antibiotika-Resistenzen in ihrer Region \\ aussieht, müssen sie selbst aktiv werden. Umfassende Informationen \\ bietet das Robert-Koch-Institut.
}
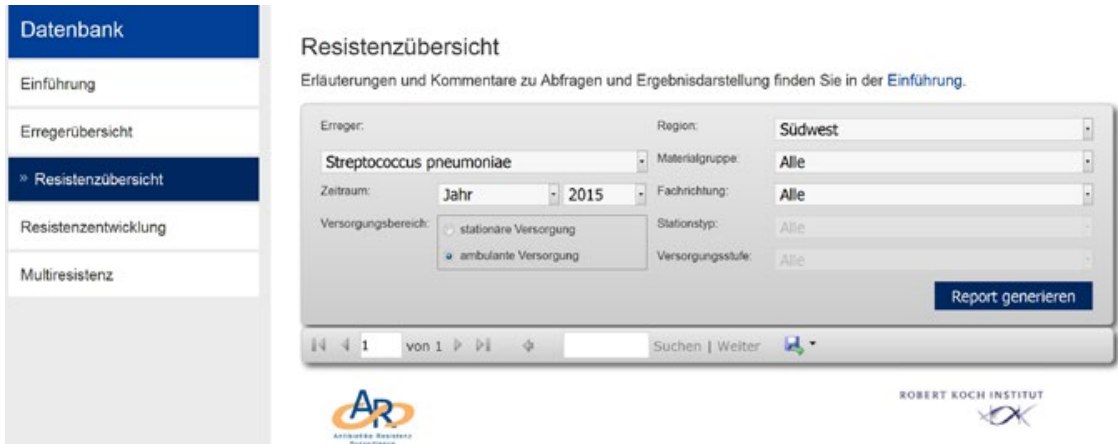

Unter ars.rki.de können Ärzte die Antibiotika-Resistenz-Surveillance (ARS) durchsuchen.

$\mathrm{M}$ ittlerweile steht das Thema Antibiotika-Resistenzen sogar auf der Agenda der großen Politik. Beim G7-Gipfel auf Schloss Elmau wurde darüber diskutiert, und das Bundeskabinett hat im Mai die neue deutsche Antibiotika-Resistenz-Strategie (DART 2020) verabschiedet. Darin sind mehrere Ziele genannt, die für Ärzte in Praxis und Klinik relevant sind:

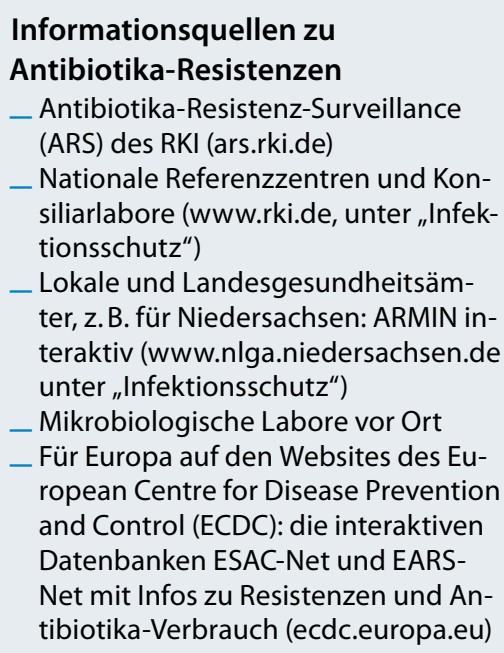

_Überwachung der Antibiotika-Resistenzen auf lokaler Ebene,

- Ausbau des Feedback-Systems für antibiotikaverordnende Ärzte und _eine internetbasierte Plattform.

Das ist jedoch eher Zukunftsmusik. Aktuell sind Informationen zu AntibiotikaResistenzen bei der Antibiotika-Resistenz-Surveillance (ARS) des RobertKoch-Instituts abrufbar (ars.rki.de). Diese Datenbank ermöglicht einen Überblick über die Resistenzen und deren Entwicklung. In der Abfragemaske „Resistenzübersicht" muss der Nutzer nur den Erreger auswählen, zwischen ambulanter und stationärer Versorgung wählen und auf den Button „Report generieren“ klicken. Neben einer Liste mit Antibiotika erhält man daraufhin auch Infos darüber, wie viele getestete Erregerisolate sensibel, intermediär und resistent sind.

\section{Auswahl nach fünf Regionen möglich}

Als Option kann dabei nach fünf Regionen in Deutschland unterschieden werden. Obwohl es möglich ist, mehrere optionale Parameter zu kombinieren, wird dies nicht empfohlen, da mit jeder Auswahl die Anzahl der Isolate, die in die Berechnung eingehen, kleiner wird. Ergibt die Auswahl weniger als 50 Isolate, werden keine Ergebnisse angezeigt.

Möglicherweise kritisch ist, dass die Daten zum Erreger mit sämtlichen getesteten Antibiotika gelistet werden. Daher erscheinen auch Reserve-Antibiotika wie die Carbapeneme. Letztlich obliegt es also dem Arzt, für seinen Patienten das jeweils passende Antibiotikum auszuwählen. Die Handlungsmaxime "alt vor neu" dürfte eine gute Richtschnur sein, solange eine ausreichende Sensibilität des Erregers vorliegt.

Diese Information wiederum kann das ARS liefern. So sind etwa Pneumokokken (Streptococcus pneumoniae) in Deutschland empfindlich gegen Penicilline. Die S3-Leitlinie zur Behandlung von Erwachsenen mit ambulant erworbener Pneumonie (AWMF-Registernummer 020-020) empfiehlt daher auch, bei leichter Pneumonie ohne Komorbidität die antimikrobielle Therapie mit einem hochdosierten Aminopenicillin zu beginnen.

\section{Neues System der Verantwortlichkeit} könnte umgesetzt werden

Was die Integration von Datenbank und Leitlinien angeht, dürfte für die Zukunft zu überlegen sein, Elemente der sogenannten Antibiotic Stewardship (ABS) einzubauen. In Kliniken mit diesem Programm nennen die Labore bei mikrobiologischen Tests nur noch zwei bis drei infrage kommende Antibiotika. Die komplette Liste aller getesteten Substanzen wird nicht mehr an die Behandler übermittelt. So soll niemand in Versuchung geführt werden, Antibiotika einzusetzen, die besser in Reserve gehalten werden.

Letztlich braucht es ein Push-System, das aktuelle Informationen quasi automatisiert in die Praxen bringt. Was hier die Umsetzung der DART 2020 bringt, wird die Zukunft zeigen. Dr. Michael Hubert 